EDITORIAL

\title{
Publicación científica en era digital y pandemia: mirada a publicaciones en la Revista Médica Hondureña
}

\author{
Scientific publication in digital era and pandemic: glance at the Revista Médica Hondureña publications
}

Actualmente los avances tecnológicos ejercen una influencia creciente y transformadora en todos los niveles de la práctica, educación y publicación en salud, siendo especialmente catalizados por la pandemia de COVID-19. Estos avances incluyen aspectos de lo que se denomina medicina digital, ingeniería sanitaria, inteligencia artificial, aprendizaje automático, acceso abierto, datos abiertos, políticas y prácticas informadas en la evidencia, entre otros, que en su conjunto engloba la llamada revolución digital o tecnológica. ${ }^{1}$ Bajo este marco contextual, la Organización Mundial de la Salud (OMS) está impulsando una estrategia mundial de salud digital con la visión de "mejorar la salud para todos, en todas partes, acelerando el desarrollo y la adopción de soluciones de salud digital apropiadas, accesibles, asequibles, escalables y sostenibles centradas en la persona para prevenir, detectar y responder a epidemias y pandemias, desarrollando infraestructura y aplicaciones que permitan a los países utilizar los datos de salud para promover la salud y el bienestar, y para alcanzar los Objetivos de Desarrollo Sostenible relacionados con la salud."

Por otra parte, también se ha reconocido que, aunque la revolución digital está cambiando el sistema global de información en salud, en la primera línea de atención la realidad ha cambiado relativamente poco. La iniciativa Información en Salud para Todos (Healthcare Information for All, HIFA, https://www.hifa.org/), ${ }^{3}$ señala que miles de personas continúan muriendo innecesariamente todos los días debido a la falta de intervenciones para salvar vidas. Aunque algunas intervenciones están disponibles localmente, no se brindan debido a la falta de información en salud, lo que resulta en indecisión, retrasos, diagnósticos erróneos o tratamientos incorrectos en todos los niveles, desde el hogar hasta los establecimientos de salud. HIFA ha propuesto recientemente una estrategia para acelerar el progreso hacia el acceso universal a información en salud fiable la cual presenta siete cambios estratégicos: convocar a las partes interesadas, fortalecer la colaboración con OMS, promover el multilingüismo, identificar y abordar las cuestiones prioritarias, aprovechar la inteligencia colectiva, fortalecer la promoción y protegerse de la desinformación. ${ }^{3}$

La desinformación y el exceso de información (infodemia) han impactado negativamente en las acciones globales para responder a la pandemia. La Secretaría de Asuntos Educativos y Culturales del Colegio Médico de Honduras implementa actividades de educación médica continua y promueve el uso de sitios validados como la Biblioteca Virtual en Salud (www.bvs.hn) para mitigar estos efectos negativos. ${ }^{4}$ Durante este período, la Revista Médica Hondureña ha publicado cuatro números y tres suplementos resultando en una publicación cada 3.4 meses en el periodo enero 2020-diciembre 2021. Del total de 80 artículos publicados (Volúmenes 88 y 89 ), 19 (24\%) se enfocaron en COVID-19, siendo la mayoría editoriales (8), seguidos por artículos de opinión (6), revisión narrativa o artículo especial (3), imagen en la práctica clínica (1) y artículo original (1). La temática abordada es variada, desde el punto de vista de salud pública y aspectos epidemiológicos incluyendo la preparación hospitalaria y desastres ecológicos hasta aspectos clínicos como neumonía y enfermedades cardiovasculares, protocolos de manejo y profilaxis, además de temas generales como prioridades de investigación, conmemoración de aniversarios y tributo a los médicos fallecidos. Los artículos publicados manifiestan la escasez de información original como estudios de investigación y casos clínicos, lo cual es un reflejo de la debilidad del ecosistema de investigación para la salud en Honduras. Es necesario fortalecer dicho ecosistema en todos sus componentes para hacer frente a los problemas sanitarios que van más allá de la pandemia, desde problemas estructurales hasta disponibilidad de insumos y materiales, formación de talento humano, manejo de datos y aspectos éticos de la investigación. La investigación, como una función esencial, ${ }^{5}$ debe integrarse operativamente al sistema sanitario.

Jackeline Alger, MD, PhD @https://orcid.org/0000-0001-9244-0668 Directora

Revista Médica Hondureña

Primera vez publicado en línea 14-12-2021

Dirigir correspondencia a: jackelinealger@gmail.com

DECLARACIÓN DE RELACIONES Y ACTIVIDADES FINANCIERAS Y NO FINANCIERAS: Ninguna.

DECLARACIÓN DE CONFLICTOS DE INTERÉS: Ninguno.

Forma de citar: Alger J. Publicación científica en era digital y pandemia: mirada a publicaciones en la Revista Médica Hondureña. Rev Méd Hondur. 2021; 89(2): 87-88. DOI: https://doi.org/10.5377/rmh.v89i2.12966

(C) 2021 Autor(es). Artículo de acceso abierto bajo la licencia https://creativecommons.org/ licenses/by/4.0/deed.es

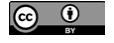




\section{REFERENCIAS}

1. Naciones Unidas. Panel de Alto Nivel sobre la Cooperación Digital [Internet]. Nueva York: ONU; 2019. [citado 28 noviembre 2021]. Disponible en: https://www.un.org/es/sg-digital-cooperation-panel

2. World Health Organization. Global strategy on digital health 2020-2025. Geneva: WHO; 2021.

3. Healthcare Information for All. Press release: Healthcare Information For All (HIFA) launches new strategy to accelerate progress towards universal access to reliable healthcare information. [Internet]. Charlbury, Oxfordshire: HIFA; 2021. [citado 2 diciembre 2021]. Disponible en: https://www.hifa. org/news/press-release-healthcare-information-all-hifa-launches-newstrategy-accelerate-progress-towards

4. Navas León FJ. Educación médica continua: oportunidades de innovación en la era digital. Rev Méd Hondur. 2020:88(Supl 1): 41-44. DOI: https://doi. org/10.5377/rmh.v88iSupl.1.11678

5. Pan American Health Organization. The Essential Public Health Functions in the Americas: A Renewal for the 21st Century. Conceptual Framework and Description. Washington, D.C.: PAHO; 2020. [citado 2 diciembre 2021]. Disponible en: https://iris.paho.org/handle/10665.2/53124 